\title{
POLYMICROBIAL BIOFILMS AND THEIR CONTROL USING NATURAL AGENTS
}

\author{
Priya Ashrit ${ }^{1}$, Vijayalakshmi V ${ }^{2}$, Bindu Sadanandan ${ }^{3}$ \\ ${ }^{1}$ Department of Biotechnology, M S Ramaiah Institute of Technology, Bengaluru 560054 \\ ${ }^{2}$ Department of Biotechnology, M S Ramaiah Institute of Technology, Bengaluru 560054 \\ ${ }^{3}$ Department of Biotechnology, M S Ramaiah Institute of Technology, Bengaluru 560054
}

\begin{abstract}
Biofilms are microbial communities that show surface association and are enclosed in a matrix of Exopolysaccharide (EPS). It is now estimated that a significant proportion of all human microbial infections involve biofilm formation. Candida species are commensals of humans, which under stressful conditions becomes an opportunistic pathogen causing candidiasis. Biofilm formation also occurs on implanted medical devices such as stents, catheters, prosthetic materials, implants, pacemakers, endotracheal tubes etc. Biofilms in humansare often polymicrobial often consisting of Candidaand Bacteria like Staphylococcus,Streptococcus, Enterococcus, Escherichia coli, Pseudomonas aeruginosa. The clinical implications of these complex interactions between the pathogens would significant clinical impact in an immune-compromised individual. Biofilm species can exhibit antagonistic or synergistic behaviour. Research exploring polymicrobial interactions is significantdue to the failure of conventional drugs to penetrate biofilm. In such cases, herbs and spices like Garlic, Gooseberry, Clove, Cinnamon, Ginger, Turmeric, Neem, Tulsi are used as alternative therapy. These natural agents are not only cost effective but also have limited side effects. Microbial resistance is also not an issue with alternative therapy. Therefore current research focuses on identifying alternativeagents which can effectively controlpolymicrbial biofilms.
\end{abstract}

Keywords: Biofilms, Polymicrobial biofilm, Exopolysaccharide (EPS), Candida albicans, Staphylococcus,Herbs \& Spices, natural agents.

\section{INTRODUCTION}

Biofilms are microbial communities that show surface association and are enclosed in a matrix of Exopolysaccharide (EPS). It holds significance since biofilms are involved in significant proportion of all human microbial infections. Candida species as commensals in humans mostly encounter with implanted devices and also host tissues. Biofilm formation occurs on devices such as stents, urinary catheters, pacemakers, endotracheal tubes (Kokare et al., 2009). Biofilms are composed of different species of microbial cells and extracellular polymeric matrix (EPS). Production of this matrix is affected by the nutrients of growth medium, where in excess amount of carbon and limited amount of nitrogen and potassium encourage EPSproduction (Ramage et al., 2005). Biofilm associated organisms are also different from their planktonic microorganisms with respect to the genes that are transcribed (Donlan et al., 2002). Biofilms are composed of different types of microorganisms such as protozoa, bacteria, algae and fungi, where each microorganism perform specialized metabolic functions. Biofilm shows pathogenicity and virulence (Kokare et al., 2009).

Polymicrobial nature of biofilms houses members of the endogenous microbiota as well as nosocomial pathogens. Polymicrobial biofilm often consists of multiple microbial species such as fungi mainly various species of Candida viz C.albicans, C. glabrata, C.tropicalisand bacteria like
Staphylococcus,Streptococcus, Enterococcus, E.coli, P.aeruginosa. Various interactions within mixed-species consortia like competition, commensalism, mutualism, parasitism etc leads to the improved survival of microorganisms within polymicrobial biofilm. The clinical implications of these complex interactions between the pathogens would significant clinical impact in an immunecompromised individual(O'Donnell et al., 2015).

Biofilm species can exhibit both antagonistic effect as well as synergistic relationship. Physical, chemical and biological parameters influence biofilm formation. Many nosocomial chronic infections are attributed to the persistence of polymicrobial biofilms. Research exploring these fungalbacterial interactions in a polymicrobial biofilm is increasing due to antimicrobial resistance and also the failure of conventional drugs to penetrate biofilm. In this situation, natural antimicrobial agents can act as potential alternative therapies to control the growth of biofilm (Peters et al., 2012).

\section{REVIEW OF LITERATURE}

Biofilm are communities of various microbes that are attached to a biotic or an biotic surface and enclosed in selfproduced matrix composed of DNA, proteins, polysaccharides. Cells present within the biofilm are different from their planktonic counterparts. According to epidemiological studies biofilm formation causes more than 
$60 \%$ of human infections. The presence of multiple species within a biofilm has resulted in organisms developing resistance to available antimicrobial agents and host's immune system. Therefore prevention and control of biofilm formation is necessary by applying effective methodologies. (Harriott et al., 2009; Klotz et al., 2007).

\subsection{Biofilm Formation}

Biofilms formation occurs in various steps. Individual cells attach to the surface which is pretreated with suitable macromolecule. Cells are absorbed on surface and cell cell signalling molecules are produced, substrate is transported to and within the biofilm which is utilized by the biofilm bound cells and products are transported out of the biofilm (Mortensen 2014).

Biofilms offers several advantages to microorganisms that are attached to surface. They provide protection against phagocytosis, biocides, shear stress, dehydration and drugs, increases the risk of infection of colonized sites. Several mechanisms involved in the increased antimicrobial resistance of biofilms:

- Slow rates of diffusion of antimicrobials.

- Physiological stress like oxygen or nutrientdeprivation \&environmental stress

- Phenotypic change of the cells during phenotypic switching from planktonic forms and Quorum-sensing.

- Decreased concentration of antimicrobials intracellularly due to efflux pump expression (Bueno, 2014).

\subsection{Biofilms of Candida Species}

Candida albicans is a dimorphic fungi, an oval-shaped budding yeast found on abiotic surfaces where diverse microbes interact within the biofilm. Factors associated with virulence of Candida albicans includes dimorphic changes. Also adhesins are important in co-adhesion of Candida to tissue and host cells, molecules like HSP 90 and CR3 like receptors which regulates the immune functions of cell and hydrolytic enzymes like proteinases which increases the tissue destruction and adhesion (Morales et al., 2010; Jenkinson et al., 2012).

\subsection{Bacterial Biofilm}

Biofilm formation by bacteriaoccurs in response to various stress like environmental stress, low nutrient availability etc. Biofilms are produced by both Gram positive and Gram negative bacteria.All bacteria with exception of few produce biofilm. Bacteria commonly involved include S.aureus, S.epidermis, S.viridians, E.coli, E.faecalis, K.pneumonia, P.mirabilis and P.aeruginosa.Biofilm formation is the result of complex process involving signaling pathways.

\subsection{Polymicrobial Biofilms}

The role of polymicrobial biofilm infections in medicine is becoming more apparent. Polymicrobial biofilm consists of multiple (Xenotypic aggregates). Therefore, it is important to understand the influence of Candida and bacterial interactions inpolymicrobial biofilm communities. This helps to know how these interactions affect clinical outcomes, and to determine how we can use this knowledge to improve patient management (Douglas et al., 2003).

\subsection{Interactions in Polymicrobial Biofilms}

Bacteria and fungi are found together in a biofilm, where they interact through diverse cell signalling mechanisms. The interactions between various bacterial pathogens and fungal pathogen Candida albicans are important. For example the interaction of Candida albicans with oral bacteria is considered as a mutually beneficial interaction where as the interaction of Candida albicans with Pseudomonas aeruginosa is antagonistic and competitive in nature (Davey et al., 2000).Therefore, prevention, treatment and control of biofilm is difficult by conventional methods (Bhaskaran et al., 2015).

\subsection{Pitfalls of Conventional Antimicrobial Therapy}

Antimicrobial agents that are active against microorganisms in their planktonic forms like penicillin, tetracycline, amphotericin-B, fluconazole and nystatin apparently become ineffective when microbes form biofilms. Biofilm formers show inherent resistance to antimicrobial agents and host defense mechanisms, therefore causing many infections. Resistance has been mainly attributed to the restricted entry of the antimicrobial agents and host defensive cells into the biofilms.Antimicrobial molecules must diffuse through the biofilm matrix in order to inactivate the cells residing within biofilm. EPS acts as a diffusional barrier for these molecules by influencing both the rate of transport of the molecule interior and the reaction of the antimicrobial material with the matrix material (Donlan and Costerton, 2002). Gordonet al, 1988, in his study showed diffusion of several antimicrobial agents (ceftazidime, cefsulodin, piperacillin, gentamicin, and tobramycin) through synthetic and naturally produced alginate gels. Thus concluding that beta-lactam antibiotics showed higher rates of diffusion through the matrix as compared to aminoglycosides as these compounds wereinitially binding to alginates.

S.epidermidis and C. albicans are frequently isolated organisms from CRBSIs (Catheter Related Blood Stream Infections). The results of a study by Venkatesh etal., 2009 reported the synergistic effect of EDTA, NAC, ethanol and TLF with antibiotics nafcillin, vancomycin, fluconazole and amphotericin B against biofilms of $S$. epidermidis and $C$. albicans. Harriott and Noverr, 2009,through their study tested the sensitivity of of $S$. aureus to vancomycin in the presence of Candida biofilm matrix. The results of the study interestingly showed S.aureus showed increased resistance to Vancomycin.This can be due to delayed penetration of the drug, which provides time for S.aureus to upregulate drug resistance genes or due to EPS which may itself alter or upregulate theantimicrobial resistance gene expression of $S$. aureus, .

Therefore, prevention, treatment and control is difficult by conventional methods (Bhaskaran et al., 2015).Challenges in treatment options using conventional antimicrobial agents 
have mandated alternative routes. Therefore a search for new, more effective drugs is most essential. In this scenario, plant extracts/ natural agents can be looked up as alternative strategies as they may provide essential benefits in controlling biofilms.

\subsection{Natural Antimicrobial Agents}

From ancient times, spices, herbs and indigenous plants have been used for treating various diseases. Plants have always been traditional health folklore and have no side effects, therefore can be considered as a safe alternative. The chemical substances in the plants actually produce a definite physiological action on the human body and its medicinal value. Alkaloids, flavonoids, tannins and phenolic compounds are generally the common bioactive compounds. Most of the natural agents usually show their activity by acting as QS inhibitors that do not kill target organism, but they weaken the biofilm structure, thus exposing the organisms within biofilm to be acted upon by conventional antibiotics and the immune system (Wuet al.,2015).

Infections caused by microbes that form biofilm are difficult to treat with conventional antibiotics. Some of the natural agents like Garlic (Allium satium) exhibit antimicrobial activities due to presence of bioactive organo sulphur compounds and provide protection against fungi, virus and bacterial infections. Therefore fresh garlic extracts can be used to improve the sensitivity of multidrug resistant microbes to antibiotics (Li et al., 2015). Likewise Clove oil extracted from the cloves (Syzygium aromaticum )is used for warts, scars and acne. Several components of clove like eugenyl acetate, eugenol, 2-heptanone, methylsalicylate, methyleugenol etc., were idenified. Several studies have showed antibacterial, antifungal and antiviral activities of clove (Saeed et al., 2008). The Indian fruit gooseberry (Phyllanthus emblica) The fruit exhibit antioxidantative, antibacterial and antiviral activities. The well known antioxidants such as Catechol, ß-carotene , Ellagic acid, Indole Acetic Acid (IAA), Phloroglucinol and superoxide dismutase enzymes are present in the fruits (Nadheesha et al., 2007).

According to epidemiological studies, many phytonutrients present in the fruits provides protection against various diseases including cardiovascular disease, cancer and diabetes and also prevent the oxidative damage (Nanasombat et al., 2012). Numerous Indian household herbs and spices for example: Cinnamon, Ginger, Turmeric, Neem, Tulsi, etc likewise can serve as potential options to contol biofilm formation (Millman et al., 2013).The complications associated with the treatment of biofilm related infections are increasing alarmingly. Along these lines, potent remedial and prophylactic strategies to prevent of microbial infections due to biofilm are essential. Novel treatments and atlternative therapies are the need of the hour .The requirement for the new more secure and more successful antimicrobial agents are required.In this viewpoint, the use of herbs \& spices will not only be beneficial to health but also be cost effective and reduce the chances of side effects. Therefore efforts to identify agents that prevent cell attachment or initiate detachment from a biofilm are currently being investigated in an effort to reduce infections caused by biofilms.

\section{CONCLUSION}

Microorganisms form biofilm on contact with surfaces. Biofilm formation is associated with the production of EPS which provides homeostasis and shelter to the organisms residing within the biofilm while also acting as a diffusional barrier. Microbial biofilms in lots of cases are polymicrobial in nature housing more than one type of organism and thus adding to the complexity of such infections and their treatment. Polymicrobial biofilm infections represent a serious public health challenge due to the high mortality rate, increased cost of care, duration of hospitalization, inherent resistance to conventional antimicrobial agents and host defense mechanisms. It is thus important to understand more about polymicrobial biofilm. Research exploring these fungal-bacterial interactions ina polymicrobial biofilm is increasing due to their antimicrobial resistance, resisitance to host immune responses and also the failure of conventional drugs to penetrate biofilm. In the medical industry, efforts are being made to identify agents to reduce infections caused by biofilms either by preventing cell attachment or initiating cell detachment. In this situation, natural antimicrobial agents can act as potential alternative therapies to control the growth of biofilm in order to devise suitable measures to control biofilm and can serve as important innovative therapeutic strategies.

\section{ACKNOWLEDGEMENTS}

This work was supported by the Department of Science and Technology (DST) grant SR/WOS-A/LS-481/2013 (G). The authors wish to thank the Management \& Principal, M. S. Ramaiah Institute of Technology (MSRIT) \& M. S. Ramaiah Hospital, Bengaluru-560054, for their encouragement \& support. The authors also wish to thank the HOD, Department of Biotechnology, MSRIT for kind support \& encouragement.

\section{REFERENCES}

[1] Bhaskaran K, Nazeer, HA, Sethumadhavan K (2015) Detection of Biofilm Formation Among the Clinical Isolates of Staphylococcus aureus. Ind. J. of Appl. Res. 5(4): 620-623.

[2] Bueno J. (2014) Anti-biofilm drug susceptibility testing methods: looking for new strategies against resistance mechanism. J. Microbial Biochem Technol.: 1-9.

[3] Davey ME. and O'toole, GA (2000) Microbial biofilms: from ecology to molecular genetics. Microbiol. Mol. Biol. Rev. 64(4): 847-867.

[4] Donlan RM (2002) Biofilms: Microbial life on surfaces. Emerg. Infect. Dis. 8(9): 881-890. Perspective.

[5] Donlan RM, Costerton JW. (2002) Biofilms: survival mechanisms of clinically relevant microorganisms. Clinical microbial. Reviews 15(2):167-93. 
[6] Douglas LJ (2003) Candida biofilms and their role in infection. Trends Microbiol. 11(1): 30-36.

[7] El-Azizi, MA., Starks SE. and Khardori, N (2004) Interactions of Candida albicans with other Candida spp. and bacteria in the biofilms. J. Appl .Microbiol. 96(5): 1067-1073.

[8] Fourie R, Ells R., Swart CW, Sebolai, OM., Albertyn J, Pohl CH. (2016) Candida albicans and aeruginosa Interaction, with Focus on the Role of Eicosanoids. Front. Physiol. 7 (64): 1-15.

[9] Gordon CA, Hodges NA and Marriott C (1988) Antibiotic interaction and diffusion through alginate and exopolysaccharide of cystic fibrosis-derived Pseudomonas aeruginosa. J. Antimicrob. Chemother. 22: 667-674.

[10] Harriott MM and Noverr CM (2009) Candida albicans and Staphylococcus aureus Form Polymicrobial Biofilms: Effects on Antimicrobial Resistance; Antimicrobiol.. Agents and Chemother. 53(9): 3914-3922.

[11] Jenkinson HF and Douglas LJ (2002) Interactions between Candida species and bacteria in mixed infections. Polymicrobial diseases. ASM Press, Rock ville, USA.

[12] Klotz SA, Gaur NK, De-Armond R., Sheppard D, Khardori N, Edwards Jr, JE., Lipke PN. and El-Azizi, M. (2007) Candida albicans Als proteins mediate aggregation with bacteria and yeasts. Med Mycol. 45(4): 363-370.

[13] Kokare CR, Chakraborty S, Khopade AN and Mahadik KR (2009) Biofilms: Importance and applications; Ind. J. Biotechnol. 8: 159-168.

[14] Li G, Ma X, Deng L, Zhao X, Wei Y, Gao Z, Jia J, $\mathrm{Xu}$ J. and Sun C, (2015). Fresh garlic extract enhances the antimicrobial activities of antibiotics on resistant strains in vitro. Jundishapur J. Microbiol. 8(5): 1-6.

[15] Millman JM , Waits K, Grande H , Marks AR , Marks JC ,Price LB , Hungate AB (2013). Prevalence of antibiotic-resistant in retail chicken: E. coli comparing conventional, organic, kosher, and raised without antibiotics. F1000 Re.s.

[16] Morales DK and Hogan DA (2010) Candida albicans interactions with bacteria in the context of human health and disease. PLoS one6(4): 1-4.

[17] Nadheesha MKF, Bamunuarachchi A, Edirisinghe EMRKBM and Weerasinghe, WMSK (2011) Studies on antioxidant activity of Indian gooseberry fruit and seed. J.of Sci. Uni.Kelaniya Sri Lanka, 3: 83-92.

[18] Nanasombat S, Khanha, Phan-im J, Jitaied J, Wannasomboon S, Patradisakorn S and Wongsil A (2012) Antimicrobial and antioxidant activities of Thai local fruit extracts: Application of a selected fruit extract, Phyllanthus Emblica Linn. as a natural preservative in raw ground pork during refrigerated storage. TOJSAT. 2(1): 1-7.

[19] 'Donnell L, Millhouse E, Sherry L, Kean R., Malcolm J, Nile CJ, Ramage G (2015) Polymicrobial Candida biofilms: friends and foe in the oral cavity. FEMS. DOI: $\quad$ http://dx.doi.org/10.1093/femsyr/fov077 fov077, 1-14
[20] Peters BM. Jabra-Rizk, MA, Graeme AO, Costerton JW and Shirtliff ME (2012) Polymicrobial interactions: impact on pathogenesis and human disease. Clin. Microbiol Rev. 25(1): 193-213.

[21] Ramage G, Saville SP, Thomas DP and Lopez-Ribot, JL (2005) Candida biofilms: an update. Eukaryotic cell, 4(4): 633-638.

[22] Saeed S and Tariq P. (2008) In vitro antibacterial activity of clove against Gram negative bacteria. Pakistan J. Botany. 40(5): 2157-2160.

[23] Venkatesh M, Rong L, Raad I, Versalovic J (2009) Novel synergistic antibiofilm combinations for salvage of infected catheters. J. Med. Microbiol. 58: $\mathrm{p}$ 936-944.

[24] Wu X, Santos RR, Fink-Gremmels J. Analyzing the antibacterial effects of food ingredients: Model experiments with allicin and garlic extracts on biofilm formation and viability of Staphylococcus epidermidis. Food science \& nutzrition. 2015 Mar $1 ; 3(2): 158-68$. 